\title{
A Precise Design Method of EMI Filter Based on Compound Common-mode and Differential-mode Inductance
}

\author{
Yuwen $\mathrm{Ma}^{1}$, Chengcheng Feng ${ }^{2, *}$ Chen $\mathrm{Li}^{1}$, Lei Wang ${ }^{1}$, Zhiyi Jia ${ }^{1}$ and Jiong Chen ${ }^{2}$ \\ ${ }^{1}$ Yangquan power supply company of Shaanxi electric power company, Shaanxi 045000, China \\ ${ }^{2}$ School of Electrical Engineering, Shanghai University of Electric Power, Shanghai 200082, China \\ ${ }^{*}$ Corresponding author
}

\begin{abstract}
Based on the principle of electromagnetic interfer-ence (EMI) filter inductance, this paper proposes a composite common and differential mode inductor integrated form filter structure, design principle of common and differential mode inductor, and design equations are derived for the precise design method of EMI filter, EMI filter designed in line with the national standard. The use of interference wave spectrum analysis equipment test before and after EMI filter insertion electromagnetic interference waveform, and insertion loss measurement curve, test results analysis based on waveform, verify the filter design method is correct and the feasibility of the application of filter.
\end{abstract}

Keywords- EMI filter; common differential mode inductance integration; composite core; insertion loss

\section{INTRODUCTION}

With the development of power electronic technology, electromagnetic compatibility (EMC) has become one of the problems that electrical designers must consider. The most important and the most difficult problem to solve in EMC standard is electromagnetic interference (EMI). EMI is generally a two-way interference signal, and the interference spectrum is about $0.15 \mathrm{MHz} 30 \mathrm{MHz}$. Power line interference is the main way to incoming and outgoing equipment, through the power line, power interference can be passed to the equipment, interfere with the normal work of equipment, the same interference generated by the equipment may also be through the power line to the power grid, interfere with the normal operation of other equipment. In order to suppress the interference effectively and attenuate the interference signal to the safe range, the EMI filter module must be added into the power design. In the module design of EMI filter, filter inductor as main filter, its performance quality directly determines the overall performance of the filter ${ }^{[1]}$, filter circuit part with common mode inductance, leakage inductance are generated by common mode filter differential mode interference, it is difficult to achieve better filtering effect; While adding two kinds of filter inductance and filter design will increase the cost, according to the principle of common and differential mode filter inductor, introduces a composite type common and differential mode inductor EMI filter structure, and combining with the design method of EMI filter in general, through the analysis of electromagnetic interference of the circuit in general, common mode interference and difference mode interference, clear interference characteristics and the required amount of interference suppression ${ }^{[2]}$, respectively establish the common mode interference filter model and differential mode interference filter model, select the appropriate filter parameters, designed to meet the needs of the EMI filter, and the experimental results verify the feasibility of filter design method.

Recently, some engineers to conduct a thorough study on the common mode and differential mode inductance structure optimization, and puts forward several simple and practical common-mode/differential-mode integration filter inductor design method ${ }^{[3,4]}$, there are still some defects. This paper proposes and designs a double magnetic shunt common mode inductance composite structure to realize the common mode interference and the effect of filtering.

\section{PRINCIPLE ANALYSIS OF EMI}

\section{A. Principle Analysis of Compound Common Differential Mode Inductors}

Composite type double core and winding the common and differential mode inductor structure ${ }^{[5]}$ as shown in Figure 1, The use of composite coil winding method, Each inductor consists of a self-winding $N_{\mathrm{m}}$ wound around a single core and a common winding $N_{\mathrm{n}}$ wound around a double core, both of which are linearly coupled, When the common mode interference current $i_{C M}$ flows through the circuit, the magnetic flux direction formed in the double core is the same, so that the forward coupling of the coil is realized. If the self inductance of the self winding $N_{\mathrm{m}}$ and the common winding $N_{\mathrm{n}}$ is respectively $L_{m} 、 L_{n}$, the mutual inductance is $M_{C}$, the magnetic resistance in the core is $R_{c}$, and the air gap flux is ignored, the formula is:

$$
\phi_{C M}=\frac{\left(N_{\mathrm{m}}+2 N_{n}\right) i_{C M}}{R_{c}}
$$




$$
L_{C M}=L_{\mathrm{m}}+L_{n}+2 M_{c}
$$

where $L_{m}=N_{m}^{2} / R_{c}, L_{n}=2 N_{n}^{2} / R_{c}, M_{c}=N_{\mathrm{m}} N_{n} / R_{c}$, $L_{C M}$ is a common mode inductor .

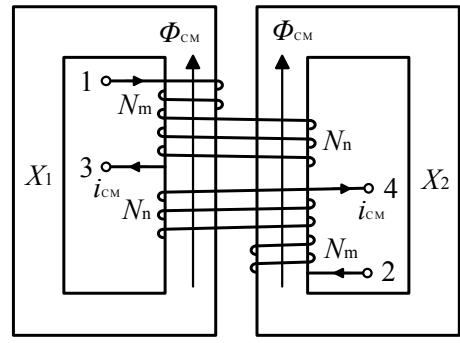

\section{FIGURE I. SCHEMATIC DIAGRAM OF COMPOSITE COMMON MODE INDUCTOR}

When there is a differential mode interference current $i_{D M}$ flowing through the circuit, as shown in Fig. 1, the magnetic flux direction formed in the double core is opposite, the formula is:

$$
\phi_{D M}=\frac{N_{\mathrm{m}} i_{D M}}{R_{c}}
$$

From the formula (3), when the differential mode current flows in the winding, the magnetic flux $\phi_{D M}$ in the two common windings $N_{n}$ is offset from each other, leaving only the self-inductance coil flux, and if the differential mode inductance is $L_{D M}$, the formula is:

$$
L_{D M}=L_{\mathrm{m}}
$$

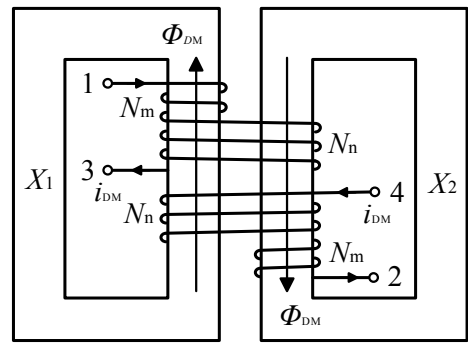

FIGURE II. SCHEMATIC DIAGRAM OF COMPOSITE DIFFERENTIAL MODE INDUCTOR

Assuming $N_{n}=k N_{\mathrm{m}}, L_{C M}=\left(2 k^{2}+2 k+1\right) L_{D M}$ is deriv-ed from the derivation of the above equation, so the common mode differential mode inductance is related to the turns ratio of the self winding and the common winding.

\section{B. Analysis of EMI Filter Structure}

Composite EMI filter basic circuit topology ${ }^{[6]}$ shown in Figure 3, the filter inductance model shown in Figure 1 and Figure 2, the filter can be common mode, differential mode interference at the same time play a decay, Figure 4 and Figure 5 are differential mode attenuation and common mode attenuation equivalent circuit structures, respectively.

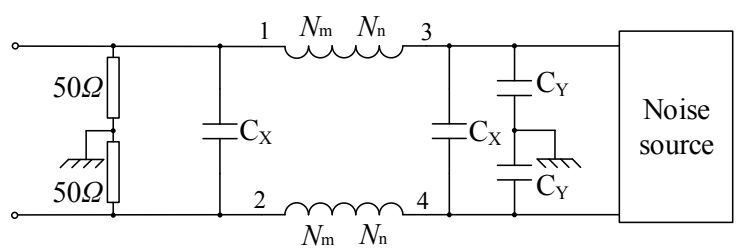

FIGURE III. THE BASIC CIRCUIT TOPOLOGY OF THE EMI POWER FILTER

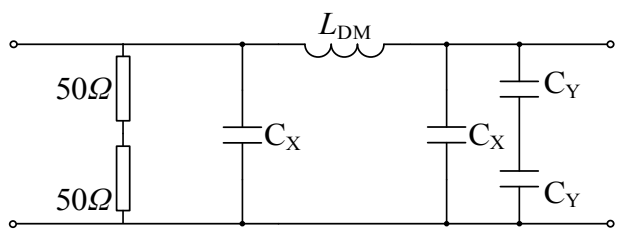

FIGURE IV. DIFFERENTIAL MODE ATTENUATION EQUIVALENT CIRCUIT

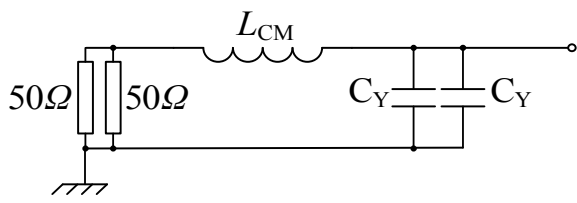

FIGURE V. COMMON MODE ATTENUATION EQUIVALENT CIRCUIT

According to the general standard, the load resistance value is $50 \Omega, L_{D M}$ is a differential mode inductor, According to the characteristics of the coil: the interference current is low when the coil impedance is low, the interference current coil frequency is high when the coil impedance is high, the interference current is determined when the high frequency using its high impedance attenuation differential mode interference signal, $C_{X}$ is a differential mode capacitor, According to the characteristics of the capacitor: the interference current frequency is low when the capacitance impedance is high, the interference current coil frequency is high when the capacitance impedance is low, the filter uses the capacitor at high frequencies when its low impedance shorts out of the differential mode interference signal. $L_{C M}$ is a common mode inductor, $C_{Y}$ is a common mode capacitor, For the differential mode attenuating circuit, the filter model is a third-order CLC low-pass filter circuit, $C_{D M}$ is the differential mode equivalent capacitance. the formula is:

$$
C_{D M}=C_{X}
$$

CLC-type filter corner frequency ${ }^{[7]}$ formula is: 


$$
f_{R \cdot D M}=\left(\frac{1}{2 \pi} \sqrt{\frac{2}{L_{D M} C_{D M}}}\right)=\left(\frac{1}{2 \pi} \sqrt{\frac{2}{L_{D M} C_{X}}}\right)
$$

For common mode attenuating circuit, the filter model is second order LC type low pass filter circuit, $C_{C M}$ is the common mode equivalent capacitance. the formula is :

$$
C_{C M}=2 C_{Y}
$$

LC- type filter corner frequency conversion formula is:

$$
f_{R \cdot C M}=\frac{1}{\left(2 \pi \sqrt{L_{C M} C_{C M}}\right)}=\frac{1}{\left(2 \pi \sqrt{2 L_{\mathrm{CM}} C_{Y}}\right)}
$$

\section{EMI FILTER PARAMETER DESIGN}

\section{A. The Main Technical Parameters}

Firstly, the EMI spectrum in the absence of the filter is measured, and the common mode interference spectrum and the differential mode interference spectrum are separated by the interference wave separation device. Calculate the attenuation of the filter within the scope of the standard, the formula is:

$$
20 \lg \left(V_{\text {req } C M}\right) d B=20 \lg \left[\left(V_{\text {measure } C M}\right)-\left(V_{\text {stan dard.CM }}\right)\right] d B+6 d B
$$

$$
20 \lg \left(V_{\text {req.DM }}\right) d B=20 \lg \left[\left(V_{\text {measure } \cdot D M}\right)-\left(V_{\text {stan dard } \cdot D M}\right)\right] d B+6 d B
$$

Where $V_{\text {req.CM }}$ and $V_{\text {req.CM }}$ are the common mode and differential mode attenuation of the filter, respectively; $V_{\text {measure.CM }}$ and $V_{\text {measure.DM }}$ are common mode and differential mode EMI respectively; $V_{\text {standard.CM }}$ and $V_{\text {standard.DM }}$ are common mode and differential mode electromagnetic interference standard, $6 \mathrm{~dB}$ for safety margin. Two common modes of slope $60 \mathrm{~dB} / \mathrm{dec}$ and $40 \mathrm{~dB} / \mathrm{dec}$ are made, and the differential dampers are tangent to the attenuation curve of the filter, The intersection of the slash and the frequency axis is $f_{R \cdot C M}$ and $f_{R \cdot D M}$. As shown in Figure 6 Figure 7 :

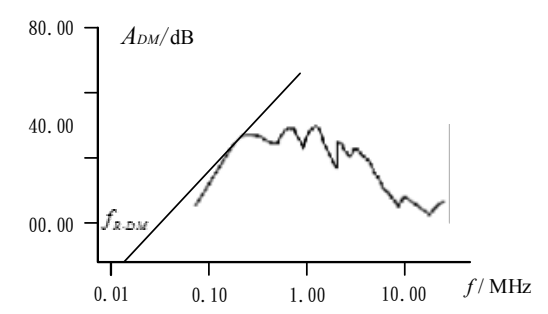

FIGURE VI. PARAMETER DETERMINATION DIAGRAM OF DIFFERENTIAL MODE FILTERING

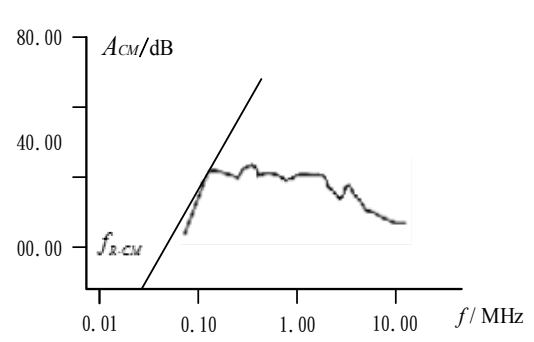

\section{FIGURE VII. PARAMETER DETERMINATION DIAGRAM OF COMMON MODE FILTERING}

The values of the required components of the filter can be calculated from the $f_{R \cdot C M}$ and $f_{R \cdot D M}$ values determined in the above article and in equations (6) and (8), As the filter inductance, the larger the value of the capacitor, the cut-off frequency is lower, the better the noise suppression effect, but the filter cost and volume are correspondingly increased, and the frequency range of the noise suppression is relatively narrow, So the value of the component can not be too big. Based on experience, $C_{X}$ ranges from 0.47 to $5 \mu F ; C_{Y}$ capacitor across the power supply line and between the ground, the ground current (leakage current $I_{g}$ ) through the common mode capacitor $C_{Y}$ out, leakage current can be calculated by the following formula:

$$
I_{g}=V_{m} \times 2 \pi f_{m} \times C_{Y} \times 10^{-6}(\mathrm{~mA})
$$

Where $V_{m}$ is the supply voltage and $f_{m}$ is the power supply frequency. As the size of the leakage current is essential for personal safety, each country on different electronic equipment grounding leakage current has strict rules, Then according to (11) can be obtained by the maximum allowable ground capacitance:

$$
C_{Y \max }=\frac{I_{g}}{V_{m} \times 2 \pi f_{m}} \times 10^{-3}(\mu \mathrm{F})
$$

$C_{Y}$ ranges from 0.02 to $0.1 \mu \mathrm{F}$.

Inductor value and material selection, the first consideration of the core material frequency range is wide, to ensure that the highest frequency $1 \mathrm{GHz}$, to ensure its broadband range has a more stable permeability; followed by high permeability, core material General use ferrite. The value of the common mode inductance $L_{C M}$ can be obtained according to equation (8):

$$
L_{\mathrm{CM}}=\frac{1}{2 C_{Y}}\left(\frac{1}{2 \pi f_{R \cdot C M}}\right)^{2}
$$

The value of the differential mode inductance $L_{D M}$ can be obtained according to equation (6): 


$$
L_{\mathrm{DM}}=\frac{2}{\left(2 \pi f_{R \cdot D M}\right)^{2} C_{X}}
$$

\section{B. Design case Analysis}

According to the above filter design method, Aiming at the EMI characteristics of Boost type active power factor correction (PFC) circuit. First, the input voltage of the PFC circuit is $220 \mathrm{~V}$, the power is $2400 \mathrm{~W}$, the switching frequency is $65 \mathrm{KHz}$, The relevant parameters of the inductive capacitance circuit can be tested with the LCR meter. Designed EMI filter related component parameters: The common mode inductance $L_{\mathrm{CM}}$ is $4.2 \mathrm{mH}$; the differential mode inductance $L_{\mathrm{DM}}$ is $6.7 \mathrm{uH}$; the common mode capacitor $C_{Y}$ is $20 \mathrm{nF}$; the differential mode capacitance $C_{X}$ is $0.47 \mathrm{uF}$. The EMI filter is inserted into the circuit, and the electromagnetic interference of the circuit is tested to obtain the common mode and differential mode interference spectrum as shown in Figure 8 and Figure 9.

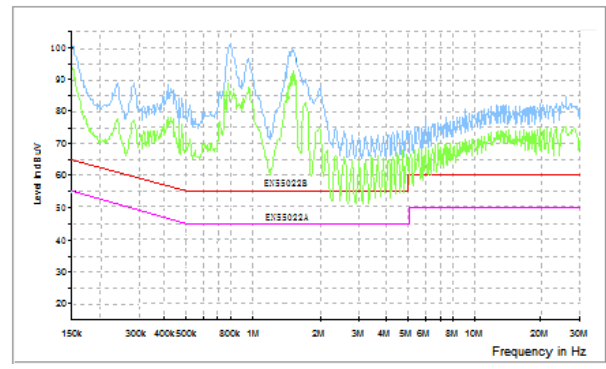

FIGURE VIII. COMMON MODE AND DIFFERENTIAL MODE INTERFERENCE BEFORE FILTER INSERTION

From the filter before the common mode and differential mode interference waveform can be seen, the interference wave beyond the standard value, must be added to the EMI filter for electromagnetic filtering.

After the filter is inserted, the common mode and the differential mode interference waveform are compared with the interference waveform before the filter is inserted. It can be seen that the filtering effect of the filter is more obvious and meets the design requirements.

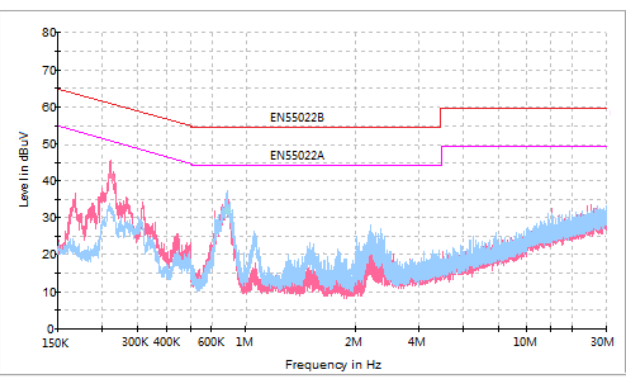

FIGURE IX. COMMON MODE AND DIFFERENTIAL MODE INTERFERENCE AFTER FILTER INSERTION

\section{CONCLUSION}

In the EMI filter design, the common mode filter inductance is the main mode for the design of composite common differential mode integrated inductors, and its choice of magnetic core mostly Mn-Zn high core material. EMI filter is proposed in this paper is the differential mode and common mode inductance composite structure and discrete filter compared to the more compact structure, smaller volume and the filter core structure, distribution parameters decreased, improve the performance, bring more inspiration for the further improvement of the structure of EMI filter.

\section{REFERENCES}

[1] Chen Kaibao , Chen Wei. Analysis of magnetic field leakage of ring common mode inductance[J]. Electrical technology, 2017, (2): 41-6

[2] Feng Cheng, Precise design of EMI filter[J] . Power Technology, 2007, (2): 119-121

[3] Jiang Shengyong, Lai Yongxue, Hu Chunyuan, Wang Hong. Common mode and common mode / differential mode integrated filter inductance magnetic saturation problem[J]. Application of Process Technology, 2011, 42(2): 62-65

[4] Wen Zhiwei, Wu Xiaofeng, Xu Dehong, Okuma Yasuhiro, Mino Kazuaki. Principle and Design of Integrated EMI Filter[J]. Journal of Electrotechnical Society, 2011, 26(9): 160-166

[5] Li Hongzhu, Zhang Lei, Wang Qiao, Qi Qingjie. A dual-core difference common mode inductor integrated EMI filter[J]. Power Electronic Technology, 2016, 50(2): 97-100

[6] Sha Zhanyou. The design principle of EMl filter[J]. Communication And Television, 2001, (5): 46-50

[7] Wu Xiaojun, Qin Kaiyu, Tang Bo. EMI filter design[J]. Electronic Test, 2011, (7): 75-81 